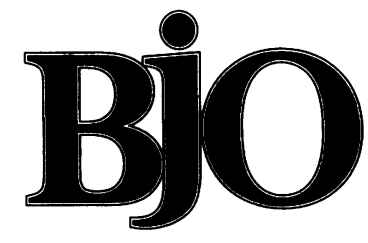

British Journal of Ophthalmology

Editorials

\title{
Prevention of postoperative infection: limits and possibilities
}

Infection of nearly every ocular structure can result in vision loss, but none is more dreaded than an intraocular infection following a commonly performed procedure such as cataract extraction. While modern methods of asepsis have markedly reduced the incidence of ocular infections in general, postoperative endophthalmitis can occur as an unexpected complication in a variety of clinical settings. Accurate statistics are difficult to obtain, but the incidence of acute postoperative endophthalmitis following cataract extraction has been reported to vary from $0.1 \%$ to $0.4 \% .^{12}$ Although the incidence is low, the potentially devastating nature of this surgical complication on useful vision merits continued effort towards both prevention and treatment.

Irrespective of the location of the infection, proper antiinfective management requires that the agents selected exhibit maximal efficacy against the causative microbes and minimal toxicity to host tissues. This basic tenet of anti-infective therapy is particularly true for intraocular infections where the eye may be saved and vision salvaged only if the treatment is prompt and vigorous. Although there is general agreement on this basic therapeutic principle, disagreement exists, often based on the experience of the surgeon, as to the best management option for prevention and treatment of infectious endophthalmitis. The therapeutic approach is further complicated by choice of antibiotic and route of administration which would effectively obliterate the micro-organism at the site of infection. $^{3}$

While the organisms most commonly associated with endophthalmitis are in the Gram positive category, Gram negative species have been isolated in $15 \%-29 \%$ of cases of clinical endophthalmitis following intraocular surgery. ${ }^{1245}$ Most studies have found Staphylococcus, including coagulase negative staphylococci, and Streptococcus species to be the most common Gram positive organisms with Pseudomonas aeruginosa, Haemophilus influenzae, and Proteus species as the most frequent Gram negative isolates. ${ }^{45}$ However, the type of infectious organisms may vary since they can be introduced into the eye by means other than surgery, such as penetrating ocular trauma and systemic infections.

Since time is usually crucial in the eventual therapeutic outcome, the antibiotic chosen should cover a broad range of organisms. Traditionally, antibiotics are selected and administered before culture results are available. Current therapy is based on broad spectrum antibiotic coverage against both Gram positive and Gram negative organisms. The classes of antibiotics most frequently used for prophylaxis and treatment of postoperative endophthalmitis have included penicillin and its derivatives, cephalosporins, aminoglycosides and, more recently, systemic fluoroquinolones. ${ }^{67}$ Commonly, aminoglycosides such as gentamicin or amikacin and the glycopeptide, vancomycin hydrochloride are administered as separate injections on an empirical basis. ${ }^{89}$ Vancomycin is commonly used since it provides protection against coagulase positive and coagulase negative staphylococcal organisms. Retinal toxicity has not been reported with intraocular injections of vancomycin in humans. ${ }^{9}$ However, irrespective of their apparent efficacy, routine intraocular injection of gentamicin and amikacin has been questioned because of potential toxic reactions. Intravitreal administration of these agents has been associated with retinal damage. ${ }^{8}$ Subconjunctival injection following cataract and other intraocular surgery causes a hyperaemic conjunctival response and myopathy of the extraocular muscles has been associated with its use. $^{10}$

Recently, the second and third generation cephalosporins have been suggested as alternatives to the aminoglycosides. ${ }^{11-14}$ The cephalosporins vary in their spectrum of activity. Second generation agents such as cefuroxime are primarily active against Gram positive organisms, whereas the third generation cephalosporins exhibit greater activity against Gram negative bacilli. Both animal and limited data in humans ${ }^{13}$ suggest that ceftazidime, a third generation drug, may be as effective as the aminoglycosides for Gram negative induced infections. ${ }^{12}$ When administered with vancomycin, limited clinical experience with ceftazidime indicates that this combination is safe and effective for prophylaxis and treatment of endophthalmitis. ${ }^{12}$

Opinions also vary as to the route of administration of anti-infective agents, particularly with more virulent cases. Topical, subconjunctival, intravitreal ocular routes, as well as systemic administration result in varying concentrations of drug in aqueous and vitreous humour. Intravitreal injections generally yield high concentrations, but this route is associated with retinotoxic effect. ${ }^{13}$ The subconjunctival route is commonly used in conjunction with intravitreal injection to enhance vitreous concentration and provide high concentrations in the anterior segment to prevent possible focal infections. The ability to deliver therapeutic 
levels of antibiotics into the eye by subconjunctival injections is supported by extensive animal data and is consistent with clinical experience in treating anterior segment infections. ${ }^{613}$

The subconjunctival route is often used for prophylactic administration of antimicrobial agents following ocular surgery. The study on comparative intraocular penetration of topical and injected cefuroxime in the current issue of the $B F O$ (Jenkins et al, p 685) indicates that subconjunctival, peribulbar, and topical application in the presence of a corneal wound can result in significant aqueous concentrations of the second generation cephalosporin, cefuroxime. Aqueous levels achieved were both dose and time dependent, with maximal concentration between 80 and 110 minutes with peribulbar injection. At this point the aqueous levels in patients given subconjunctival injections were still rising, reaching a maximum between 120 and 160 minutes. If the aqueous level of cefuroxime required for prophylaxis is equivalent to the $\mathrm{MIC}_{90}$ value of $8 \mu \mathrm{g} / \mathrm{ml}$, this was not observed with either the $25 \mathrm{mg}$ subconjunctival or $125 \mathrm{mg}$ peribulbar concentrations. However, subconjunctival injection with $62.5 \mathrm{mg}$ and $125 \mathrm{mg}$ of cefuroxime did produce aqueous concentrations which exceeded the $\mathrm{MIC}_{90}$ value. Vitreous levels of cefuroxime were not analysed but previous studies have shown negligible levels of drug penetration following subconjunctival administration. ${ }^{14} \mathrm{~A}$ previous study by Jenkins et al ${ }^{11}$ compared the effects of subconjunctival gentamicin and cefuroxime in cataract surgery. The data indicate that $125 \mathrm{mg}$ of cefuroxime results in less conjunctival hyperaemia and a better MIC value for most organisms (except Pseudomonas) than $20 \mathrm{mg}$ gentamicin. The present study did not discuss toxicity or efficacy of cefuroxime following subconjunctival administration in cataract surgery.

The question of clinical effectiveness of subconjunctival antibiotics remains a matter of conjecture. If subconjunctival administration at the end of surgery decreases the incidence of endophthalmitis, the question to answer is which drug or combination of drugs has the best risk-benefit ratio? Perhaps Leopold and Apt stated it best when they noted more than 30 years ago that prophylactic antibiotic use by subconjunctival route to prevent postoperative infections 'will be proven only as a consequence of a controlled study both instituted and performed by a single ophthalmic surgeon or surgical team' ${ }^{15}$ New possibilities may also arise from the discovery of broad spectrum systemic antibiotics, such as the fluoroquinolones, with adequate intraocular penetration to cover organisms associated with postoperative intraocular infections. ${ }^{7}$ In the meantime, the best defence against intraocular infection is skilful surgical technique, careful operative preparation, and intraocular administration of select antibiotics by rational guidelines. ${ }^{16}$

SIRET D JAANUS

State University of New York,

State College of Optometry,

New York, NY 10010, USA

1 Kattan HM, Flynn HW, Pflugfelder SC, Robertson C, Forster RK. Nosocomial endophthalmitis survey. Current incidence of infection after intraocular surgery. Ophthalmology 1991;98:227-38

Bohigian GM, Olk RJ. Factors associated with a poor visual result in endophthalmitis. Am $\mathcal{f}$ Ophthalmol 1986;101:332-41.

3 Doft BH. The endophthalmic vitrectomy study. Arch Ophthalmol 1991;109: 487-9.

Puliafito CA, Baker AS, Haaf J, Foster CS. Infectious endophthalmitis: review of 36 cases. Ophthalmology 1982;89:921-9.

5 Irvine WD, Flynn HW, Miller D, Pflugfelder SC. Endophthalmitis caused by gram-negative organisms. Arch Ophthalmol 1992;110:1450-4.

Py gram-negative organisms. Arch Ophthalmol 1992;110:1450-4. after cataract surgery. Review of 26 cases. $\exists$ Ocul Pharmacol Therapeut after cataract surger

7 Kowalski RP, Karenchak LM, Eller AW. The role of ciprofloxacin in endophthalmitis therapy. Am f Ophthalmol 1993;116:695-9.

8 Campochiaro PA, Conway BP. Aminoglycoside toxicity-a survey of retinal specialists: implications for ocular use. Arch Ophthalmol 1991;109:946-50.

9 Pflugfelder SC, Hernandez E, Fleisler SJ, Alvarez J, Pflugfelder ME, Forster RK. Intravitral vancomycin: retinal toxicity, clearance and interaction with gentamicin. Arch Ophthalmol 1987;105:831-7.

10 Green $\mathrm{K}$, Chapman J, Cheeks L. Ocular toxicity of subconjunctival gentamicin. Lens Eye Tox Res 1992;9:439-46.

11 Jenkins CDG, McDonnell PJ, Spalton DS. A randomised single blind trial to compare the toxicity of subconjunctival gentamicin and cefuroxime in to compare the toxicity of subconjunctival gentamitaract surgery. Br 7 Ophthalmol 1990;74:734-8.

12 Campochiaro PA, Lim JI. Aminoglycoside toxicity in the treatment of endophthalmitis. Arch Ophthalmol 1994;112:48-53.

13 Barza M, Doft B, Lynch E. Ocular penetration of ceftriaxone, ceftazidime and vancomycin after subconjunctival injection in humans. Arch Ophthalmol 1993;111:492-4.

14 Barza M, Kane A, Baum J. Ocular penetration of subconjunctival oxacillin, methicillin and cefazolin in rabbits with staphylococcal endophthalmitis. f Infect Dis 1982;145:899-903.

15 Leopold IH, Apt L. Postoperative intraocular infections. Am f Ophthalmol 1960;50:1225-47.

16 Meredith TA. Prevention of postoperative infection. Arch Ophthalmol 1991; 109:944-5. 